\title{
Modelling control options for a disease with hidden sub- clinical infection: bacterial kidney disease in Scottish aquaculture
}

\author{
$\underline{\text { A.G. Murray }}^{\text {a }}$, M. Hall ${ }^{\text {a }}$, L.A. Munro ${ }^{\text {a }}$ and I.S. Wallace ${ }^{\text {a }}$ \\ ${ }^{a}$ Marine Laboratory, Marine Scotland Science, 375 Victoria Road, Aberdeen AB11 9DB, United Kingdom \\ Email: Sandy.Murray@scotland.gsi.gov.uk
}

\begin{abstract}
Bacterial kidney disease (BKD) is a disease of salmonids that is present in Western Europe, North and South America and Japan. In Scotland BKD occurs in salmon and trout farms where it has remained at a fairly constant prevalence in spite of an official eradication policy (approximately $1 \%$ of salmon and $20 \%$ of trout farms are under official controls). The control policy has been based on movement controls on infected sites to prevent spread of infection, and fallowing to clear infection. However, the bacterium responsible for BKD, Renibacterium salmoninarum, can form sub-clinical infection. These subclinical infections can be hard to detect because diagnostic methods used have low sensitivity and within farm prevalence is often low. These subclinical infections are likely to have played an important role in undermining the eradication policy.
\end{abstract}

To investigate control options we have developed an SI model of $R$. salmoninarum where the model population consists of uninfected, susceptible $(S)$ farms and infected farms. These infected farms are subdivided into 3 categories, diseased $(D)$ and subclinical farms which consist of known $(K)$ and unknown infections $(U)$. The known infections $K$ and $D$ are subject to movement controls and so most infection spread is controlled. The unknown $U$ farms are not controlled and therefore spread infection with fish moved from these sites.

This model has been given a sensitivity analysis and used to investigate a range of management scenarios. These include: abandoning movement controls, increased surveillance, improved fallowing on all farms, and vaccination. The response to policy changes depends heavily on the level of undetected infection. Generally, salmon respond more strongly than trout to changes in controls, both positively to stricter and negatively to relaxed controls. Optimal control policies are very different for the two sectors; for trout this being the abandonment of controls and for salmon retaining or reinforcing existing controls. A new control policy has been drawn up that aims to achieve this while separating the two industries to prevent cross infection.

Keywords: Susceptible-Infected modelling, aquaculture, Scotland, bacterial kidney disease 
Murray et al., Modelling control options with sub-clinical infection: BKD in Scottish aquaculture

\section{INTRODUCTION}

Aquaculture production in Scotland has become increasingly important to the economy. It is an important source of employment and investment in remote regions of western and island Scotland. The majority of production is of Atlantic salmon (Salmo salar); Scotland is the world's $3^{\text {rd }}$ largest producer of farmed Atlantic salmon at 144,247 tonnes in 2009 (Walker 2010). Farm gate value is estimated at £500M for 2010 and constituted Scotland's largest single food export product. At 6766 tonnes the production of rainbow trout (Oncorhyncus mykiss) is also significant.

Diseases can have a negative impact on aquaculture production, an analysis of a Scottish database found about a third of salmon mortality is associated with disease (Soares et al. 2011), and large outbreaks can be highly disruptive. Control and eradication of diseases are thus desirable to improve fish production and welfare; however disease control policies such as movement controls can be costly to industry, so they must be effective to be justifiable.

One disease that is considered to be a significant cause of losses to infected Scottish salmon farms is Bacterial Kidney Disease (BKD). This disease of salmonids is also present in Western Europe, North and South America, Chile, Japan (Austin and Austin 2007). The United Kingdom was granted additional guarantees by the European Union, allowing control of imports of live fish and ova but requiring an eradication programme (Munro 2007). This programme placed infected sites under designated area orders (DAOs) which imposed movement restrictions on the site. These movement restrictions are now called Confirmed Designation Notices (CDNs), however we use the term that applied at the time to which the model is fitted. The system appeared quite effective at keeping prevalence low for salmon with (at any one time) $0-2.5 \%$ of sites under DAOs, but about $15-20 \%$ of trout farms were under DAOs from 2004-8. However, there was little evidence of progress towards eradication, meaning import controls were not legally sustainable. Furthermore, although hard data are limited, the experience of trout farmers was that most farms with BKD suffered little additional mortality, while salmon farms could suffer significantly increased mortality. The trout industry was paying the heaviest control costs while the salmon industry was benefiting most from this control policy.

The causal agent of BKD is Renibacterium salmoninarum; this bacterium can also cause subclinical infection. These subclinical cases can be very hard to detect (no passive detection, poor diagnostic test sensitivity, often low farm-level prevalence). This means infected (but not diseased) sites may go undetected and hence targeted movement controls cannot be imposed on these sites. However, as movements generally involve many thousands of fish, even low prevalence pathogens are likely to be included in any individual movements.

The failure of eradication, the unfair cost:benefit distribution between salmon and trout and the poor quality of surveillance meant that the BKD control policy had to be reviewed. Why was eradication not occurring and could this be achieved? If not, was there an alternative policy that allowed BKD to continue to be managed at the low level found in salmon while not imposing excessive controls on trout? To assess the policy we reviewed the recent history of BKD in Scotland (Murray et al. 2011) and used this information to develop a susceptible-infected population model that describes our understanding of the epidemiology and management of BKD in Scotland. This model is used to test alternative control polices.

\section{THE MODEL}

The approach we have used to model the dynamics of BKD is based on the classic susceptible-infected approach of Anderson and May (1979). In this model our population is the farms in the industry (salmon, trout or both) divided between the proportions of farms that are susceptible (S), or are infected (Fig. 1). The infected farms are subdivided between the proportion of farms known to be infected $(K)$, the proportion that are infected but unknown $(U)$ and the proportion of farms that are diseased $(D)$ - since BKD is notifiable the presence of disease are assumed to be officially known. The model does not look at farm level prevalence, so if $0.5 \%$ or $50 \%$ of fish on a farm are infected then that farm is classed as infected.

The observed prevalence of DAOs has been fairly constant at about $1 \%$ of salmon farms and $20 \%$ of trout farms so it is reasonable to treat the model as being in steady state.

Infection is transmitted from infected farms to susceptible farms by the movement of fish and to a lesser extent through the environment. In simulation of the existing policy we assume movement controls are 
Murray et al., Modelling control options with sub-clinical infection: BKD in Scottish aquaculture

imposed on farms known to be infected ( $K$ and $D$ ) so movement-based transmission occurs only from $U$. Transmission through the environment is limited (Austin and Rayment 1985, Murray et al. 2011) and we assume that this is negligible from non-diseased farms, so such transmission only occurs from $D$. We use an infection transmission formulation of the form $\beta S I$ (Anderson and May 1979). Transmission is density independent because the model variables are the proportions of farms of different infection status. By using an arbitrary time-step we can set the transmission coefficient $\beta$ to an arbitrary value $b$ that applies when transmission is uncontrolled. We use different transmission coefficient parameters of $\beta_{1}=b$ from $U, \beta_{2}=$ 0 from $K$ and $\beta_{3}=0.2 b$ from $D$, allowing for some environmental transmission. In practice we use $b=0.2$, as a value that provides numerical stability while being computationally efficient.

Infected farms of any status can lose infection perhaps as a result of fallowing, these may be at different rates since more aggressive fallowing may be carried out at known infected or diseased farms, the parameters are $n_{1}, n_{2}$ and $n_{3}$. Infected farms ( $U$ or $K$ ) can develop clinical disease $(D)$ at a rate $x$, and disease can resolve leading to



Figure 1. The BKD modelling structure. Red $D=$ diseased farms; yellow are farms that are infected with $R$. salmoninarum but do not have BKD ( $K=$ known, $U=$ unknown infection); green $S$ are uninfected, susceptible, farms. farms reverting to sub-clinical infection at a rate $r$. Sites losing disease but retaining infection revert to $K$ status as the presence of infection is known from the disease history.

Technically, $U$ farms can become $K$ as a result of routine surveillance. However, assessment of the history and sensitivity of the surveillance regime of the time indicates detection is unlikely (Hall et al. 2011) - we do include a parameter $q$, set to zero by default, to investigate scenarios of improved surveillance. However, targeted surveillance using contact tracing based on movement histories in an out of sites that develop BKD does identify cases. We therefore include a contact tracing parameter $c$ which is multiplied by the rate of onset of disease in sites not known to be infected $(x U)$.

The model equations are thus:

$$
\begin{aligned}
& d S / d t=-\beta_{1} S U-\beta_{2} S K-\beta_{3} S D+n_{1} U+n_{2} K+n_{3} D \\
& d U / d t=\beta_{1} S U+\beta_{2} S K+\beta_{3} S D-U\left(c x+n_{1}+x+q\right) \\
& d K / d t=r D+c x U+q U-x K=n_{2} K \\
& d D / d t=x U+x K-n_{3} D-r D .
\end{aligned}
$$

The observed prevalence of BKD has been fairly steady in Scotland, as a result it is reasonable to assume the epidemic is at steady state. If this is the case, then the rate of removal of infection is in balance with the rate of formation of infection so we can calculate this rate, given assumed values for the steady state value of unknown infection $\left(U^{*}\right)$, and how the known number of sites with DAOs is split between $K^{*}$ and $D^{*}$, (we assume equally split, but the model has low sensitivity to the exact value used). We split the removal rate between $U, K$ and $D$ using a parameter $y$, by which removal of known infection $(K$ and $D)$ is faster than removal of unknown infection $\left(n_{2}=n_{3}=y n_{1}\right)$. It is possible $y=1$ if there is no special management of known 
Murray et al., Modelling control options with sub-clinical infection: BKD in Scottish aquaculture

infected sites, however we assume $y=2$ for the default value. Having evaluated these parameter we can go on to evaluate $x$ and $r$ by similar assumption of steady state.

The model is left with a minimal parameter freedom, the value of $y(=2), c(=1)$, the additional transmission through the environment $(=0.2 b)$, the split of the sites under DAOs between $K^{*}$ and $D^{*}$ (we assume 50/50) and the proportion of farms with unknown infection at steady state, $U^{*}$. Note the value of $b$ is arbitrary, and so does not need to be explicitly evaluated, however this arbitrariness means that we do not know the time scale over which temporal responses occur in the model and so do not know their significance. The model output is not sensitive to the assumed values of these, except to $U^{*}$, to which its behaviour is highly sensitive. We do not know the prevalence of undetected infection, except that it is less than $1-(K+D)$. We thus must explore model outputs under a large range of possible values of $U^{*}$.

\section{MANAGEMENT POLICIES FOR BKD}

\subsection{Scenarios}

Having set the model to existing conditions, it can be used to explore BKD's response to new management scenarios by appropriate changes to parameter values, assuming a range of possible values of $U^{*}$. Results are displayed for prevalence of known infection in salmon $\left(K^{*}=D^{*}=0.005\right)$ and trout $\left(K^{*}=D^{*}=0.1\right)$ and with the model parameter evaluated to produce these steady states. This modelling of salmon and trout as separate compartments is reasonable given the very different BKD prevalence in these industries and because of epidemiological separation (see section 3.3) Here we present potential changes to the status quo; either abandoning existing movement controls or finding alternative ways of tightening controls.

Since BKD controls have not lead to eradication, one response could be to lift the movement controls on infected sites, i.e. set $\beta_{2}=\beta_{1}$ and $\beta_{3}=1.2 \beta_{1}$, as the $20 \%$ transmission rate that occurs through the environment from sites with clinical disease will continue to occur around infected sites when movement controls are lifted.

Alternatively, restrictions could be tightened with the aim of eradication or at least improved control. There are several ways this can be done and we illustrate here with the examples of improved fallowing, improved surveillance, and vaccination. Other strategies such as culling of infected sites and stricter movement controls on all sites have also been investigated.

We investigate improved fallowing on all sites regardless of infection status, simulated by doubling $n_{1}, n_{2}$ and $n_{3}$. This scenario implies that the same practices are undertaken at uninfected sites $(S)$ as at unknown $(U)$ sites since these cannot be distinguished.

We could also improve surveillance, quantitative polymerase chain reaction (qPCR) for example has the potential to be much more sensitive at detecting subclinical $R$. salmoninarum than the enzyme-linked immunosorbent assay (ELISA) screening used up to 2010 (Hall et al. 2011). We simulate this in then model simply be increasing q from zero to a finite value we illustrate this with a value of $0.1 b$ as a possible scenario. This could be achieved in reality by increased sample numbers and/or sizes or by using a more sensitive diagnostic method. Our work suggests use of qPCR could achieve a very large increase in sensitivity for detection of subclinical infection over the method of ELISA confirmed by culture used at the time.

Vaccines for BKD are poor and are not used in Scotland, but recent developments suggest it may be possible to reduce $\mathrm{BKD}$, but not to eradicate $R$. salmoninarum by vaccinating the population. Vaccinated populations may become infected but fail to develop disease and thus remain in $U$ status. This is simulated by reducing $x$, the parameter for rate of onset of disease, by $50 \%$.

\subsection{Results}

If movement controls are lifted then infection can be spread by movements off sites currently under DAOs $(K$ or $D$ ). The result is more potential $R$. salmoninarum spread and so more cases of BKD (Fig. 2). We divide the prevalence of disease at the new steady state $\left(D^{+}\right)$by the prevalence before the policy change $\left(D^{*}\right)$ and deduct 1 to derive the relative change in cases of BKD (change $\left.=\left[D^{+} / D^{*}\right]-1\right)$. The change is highly sensitive to the existing steady state proportion of sites with undetected infection $\left(U^{*}\right)$ that are not under controls 
anyway. If $U^{*}$ is high the effect of abandoning controls is minimal, but if it is low then BKD cases increase substantially, potentially by a factor of 2-3 in the case of trout and by an order of magnitude for salmon. While we do not know the value of $U^{*}$, we do know how many known cases of infection exist. If unknown cases are proportional to known sites with DAOs then we expect $U^{*}$ to be low for salmon, but perhaps moderately high for trout. If so, the order of magnitude increase in salmon cases and a much lower increase for trout is likely; remember BKD also inflicts higher losses-per-case on salmon than on trout. It thus seems trout farms are gaining relatively little benefit, and are disproportionately subject to the controls relative to salmon. Therefore salmon farmers have more to gain from maintaining controls.

Trout also respond less vigorously to strengthened controls than salmon in terms of the impacts of BKD (Fig. $3)$. Three controls are illustrated: improved fallowing on all sites; improved surveillance $(q=0.02)$; and vaccination $(0.5 x)$. These scenarios have rather different impacts and so are illustrated with different outputs.

Improved fallowing leads to the eradication of BKD from salmon farms for most cases, and a large decline for trout but not eradication, unless $U^{*}$ is very low (Fig. 3A). Reinfection of cleared sites, particularly if $U^{*}$ is large, may mean that fallowing only slowly removes BKD from the industry-level population. Salmon farms already fallow to a high standard after every production cycle, so improving on this may not be practicable. However, some trout farms have not been regularly fallowed, so it is possible that control through improved fallowing would actually be more practicable in trout (Wallace et al. 2011).

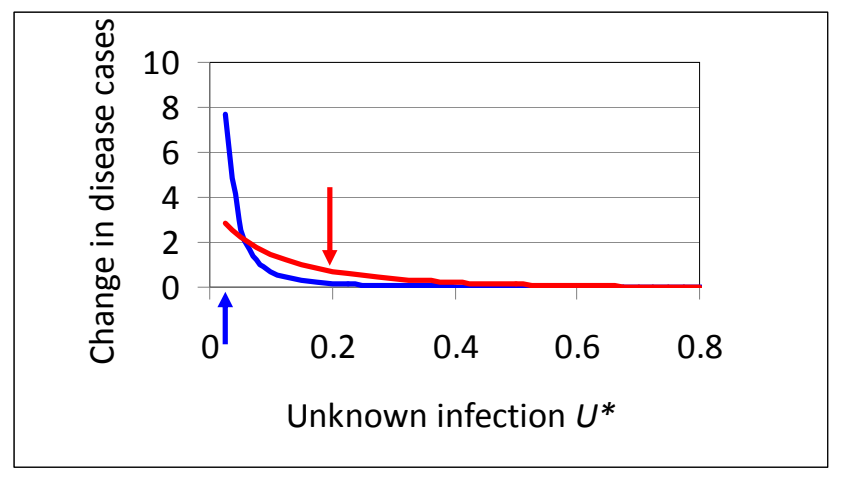

Figure 2. Relative change in BKD prevalence on lifting controls on movement (blue $=$ salmon, red $=$ trout) versus unknown infection. Arrows indicate prevalence of DAOs (known infection)
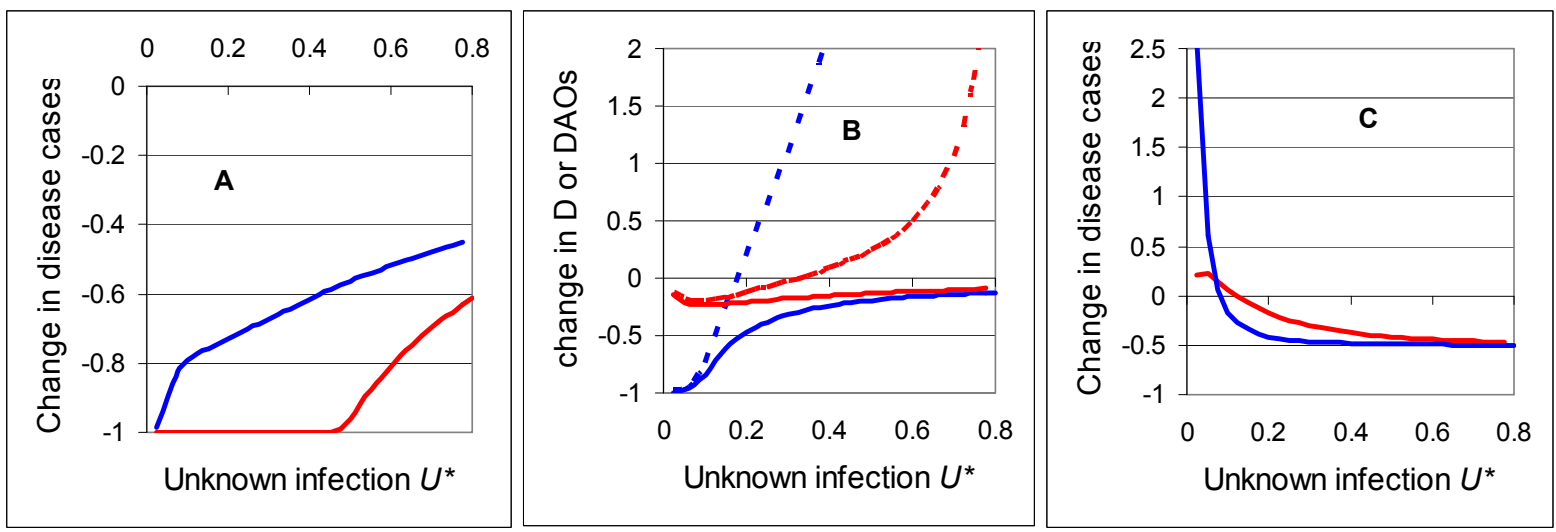

Figure 3. Three BKD control policies applied to trout (red) and salmon (blue) with the model run to new steady state. A. Change in relative number of disease cases when fallowing is improved on all sites regardless of infection status. B.. Change in relative number of disease cases (solid lines) and relative number of sites under movement controls (dashed lines) under improved surveillance. C. Change in relative number of cases of BKD with vaccination

Improved surveillance leads to a large drop in prevalence of disease in salmon, but has little effect on disease in trout (Fig. 3B). However, increased surveillance leads to more sites being placed under DAOs, at least in the short-term. If BKD is eradicated then the number of DAOs also becomes zero, however if $U^{*}$ is higher then the number of DAOs may be substantially increased even in the long term. This increase occurs for 
Murray et al., Modelling control options with sub-clinical infection: BKD in Scottish aquaculture

lower values of $U^{*}$ in salmon, but given the likely values of $U^{*}$ the increase in DAOs may in practice be larger for trout.

The third scenario is a vaccination policy (Fig. 3C). The vaccine is assumed to reduce onset of disease by $50 \%$, but not to suppress infection. This policy might be expected to reduce disease incidence by $50 \%$, and indeed does so if $U^{*}$ is large. However, if $U^{*}$ is small the effect of vaccination can be counter-productive, with a small loss of efficacy for trout but for salmon there is a possible collapse of controls. The reason for this failure is that vaccination masks sub-clinical infection and so undermines movement controls. The losses suffered might be less that this implies, because losses per diseased farm could well be less if these farms use vaccination.

\subsection{Separate management of farmed salmon and trout}

The response to changes in management practices suggests there are different optimal strategies for salmon and trout, and that salmon were disproportionately benefiting from the existing controls. Marine Scotland Science (MSS) therefore reviewed the structure of the respective industries to investigate the possibilities for implementing separate management strategies (Murray et al. 2011). In Scotland there is a high degree of physical separation of salmon (western and northern) and trout (southern and eastern) producing areas (Fig. 4). Potential epidemiological risks were highlighted in a few areas and these required a more in depth analysis. Region I, Shetland, is an area with trout and salmon farms, and has had cases of BKD in salmon. However, the trout use local broodstock and do not appear to have been involved. In the Western Isles (II) salmon and sea-farmed trout are held on the same sites, again local broodstock are used, there have been no cases of BKD. Region III contained a large trout farm within

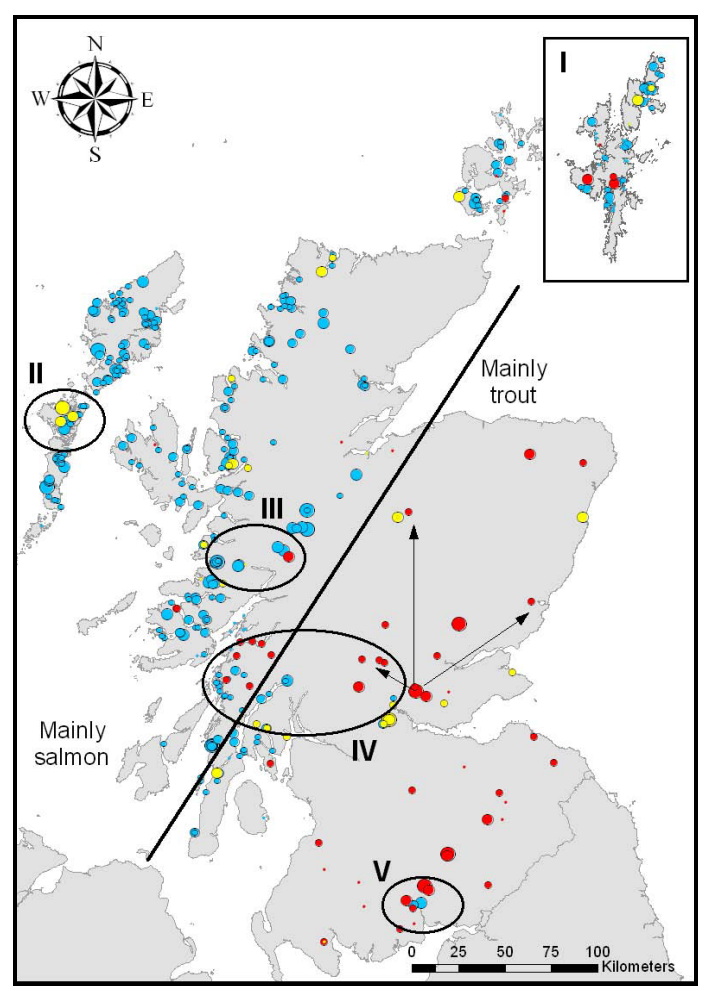

Figure 4. Distribution of salmon (blue), trout (red) and mixed (yellow) farms in Scotland. Farm circle size is proportional to movements on and off farms. Areas I to V are discussed in text. a salmon farming region. This showed statistical evidence of association with BKD in salmon; following its closure there have been no cases in the local salmon farms. Region IV in the southwest Highlands is a large area with salmon and rainbow trout farms and BKD has occurred in the trout. However, salmon and trout farms are generally in different catchments or separated by impassable natural barriers. In region $\mathrm{V}$ salmon farms located in a trout producing area, use secure borehole-water and so are not exposed to the trout farms.

It appears there is only limited contact between salmon and trout farming areas illustrated in Fig. 4. An analysis of the contact network structure showed that catchments in the salmon and trout farming regions are indeed separate. There are 3.85 intra-regional contacts per site within the salmon region and 3.31 for within the trout region but only 0.17 contacts per site for movements from the trout to salmon regions.

Prevalence of $R$. salmoninarum in wild fish in Scotland is extremely low with only very small numbers having given positives results by qPCR during research sampling, and none since the 1960s from standard wild fish screening with ELISA (Wallace et al. 2011). This contrasts with the 1930s when BKD occurred as a clinical disease of wild salmon (Dee disease). It is possible that wild or escaped fish might act as a reservoir, but it seems unlikely they could act as a significant vector transporting infection between farms. 
Murray et al., Modelling control options with sub-clinical infection: BKD in Scottish aquaculture

\section{A NEW CONTROL POLICY}

Following a review by MSS and extensive discussion involving government, the British Trout Association and the Scottish Salmon Producers Organisation a policy was agreed that met the needs of both industries (Richards 2011). Under this policy background surveillance, which was held to be ineffective (Hall et al. 2011) was discontinued, however BKD remained notifiable. If suspicion of BKD was reported the farm would be officially inspected and if signs of BKD were present fish would be screened using the more sensitive qPCR. Contact tracing around infected sites would result in inspection of sites that shared a catchment (or sub-catchment only in larger systems) or had received or delivered fish to known infected sites. Only if moribund fish were observed would a diagnostic sample be taken. If clinical BKD was diagnosed movement restrictions would be imposed. These prohibit the movement of fish or ova to catchments or marine management areas that hold salmon farms, but not to catchments that include only trout farms. Restrictions on an infected farm would remain in force until the site either fallows or tests negative for $R$. salmoninarum using qPCR at the 150 fish level. The purpose of these controls are to maintain the existing low prevalence of BKD in salmon, while lifting controls on trout that do not pose excessive risk to the salmon. This also usefully reduces the cost to government of inspections.

One big uncertainty that remains is the value of $U^{*}$, and this means there is uncertainty as to the exact response of the prevalence of BKD to any specific control policy. However, the sensitivity of BKD in salmon, and the lack of sensitivity in trout are robust findings over a large range of $U^{*}$.

\section{ACKNOWLEDGMENTS}

This work has been funded by Marine Scotland using data from MSS scientists, fish health inspectors and the salmon and trout industries.

\section{REFERENCES}

Anderson R.M. and R.M. May (1979) Population biology of infectious diseases: part I. Nature 280, 361-367

Austin B. and J.N. Rayment (1985). Epizootiology of Renibacterium salmoninarum the causative agent of bacterial kidney disease in salmonid fish. Journal of fish diseases 8, 505-509

Austin B. and D.A. Austin 2007 Bacterial fish pathogens diseases of farmed and wild fish. $4^{\text {th }}$ Edition. Springer, Chichester, UK

Hall L.M., I.S. Wallace, L.A. Munro, A. Walker, A.G. Murray (2011). Epidemiology informs policy regarding surveillance of a notifiable disease of salmonids. Epidémiology Santé Animal 59-60 (Proceedings of the International Conference on Animal Health Surveillance), 392-394

Munro P.D. (2007). Implementation of additional guarantees and results of an epizootic investigation for BKD in Scotland. Fish veterinary Journal 9, 56-62

Murray A.G., L.A. Munro, I.S. Wallace, E.J. Peeler. and M.A. Thrush (2011). Bacterial kidney disease: assessment of the risk to Atlantic salmon farms from trout farms and other sources. Scottish Marine and Freshwater Science 2(3). Marine Scotland Science, Edinburgh UK. http://www.scotland.gov.uk/Publications/2011/04/21144833/0

Richards R. (2001). Bacterial Kidney Disease paper 2011. Scottish Government Ministerial Group on Aquaculture http://www.scotland.gov.uk/Topics/marine/Fish-Shellfish/mingroup/BKDPaper2011

Soares S., D.M. Green, J.F. Turnbill, M. Crumlish and A.G. Murray (2011). A baseline method for benchmarking mortality losses in Atlantic salmon (Salmo salar) production. Aquaculture 314, 7-12

Walker A.J. (2010). Scottish fish farms annual production survey, 2009. Marine Scotland - Science, Aberdeen.

Wallace I.S., L.A. Munro, R. Kilburn, M. Hall, J. Black. R.S. Raynard. and A.G. Murray (2011) A report on the effectiveness of cage and farm-level fallowing for the control of bacterial kidney disease and sleeping disease on large cage-based trout farms in Scotland. Scottish Marine and Freshwater Science 2(10). Marine Scotland Science, Edinburgh UK. http://www.scotland.gov.uk/Publications/2011/08/18155710/0 